\title{
Cross-modal Supervision for Learning Active Speaker Detection in Video
}

\section{Punarjay Chakravarty and Tinne Tuytelaars}

Abstract

In this paper, we show how to use audio to supervise the learning of active speaker detection in video. Voice Aclivity Detection (VAD) guides the learning of the vision-based classifier in a weakly supervised manner. The classifier uses spatio-temporal features to encode upper body motion - facial expressions and gesticulations associated with speaking. We further improve a generic model for active speaker detection by learning person specific models. Finally, we demonstrate the online adaptation of generic models learnt on one dataset, to previously unseen, weople in a new dataset, again using audio (VAD) We are the first to present an active speaker detection system that learns on one audio-visual and automatically adapts to speakers in a new dataset. This work can be seen as an example of how the availability of multi-modal data allows us to learn a model without the need for supervision, by ransferring knowledge from one modality to another. Applications

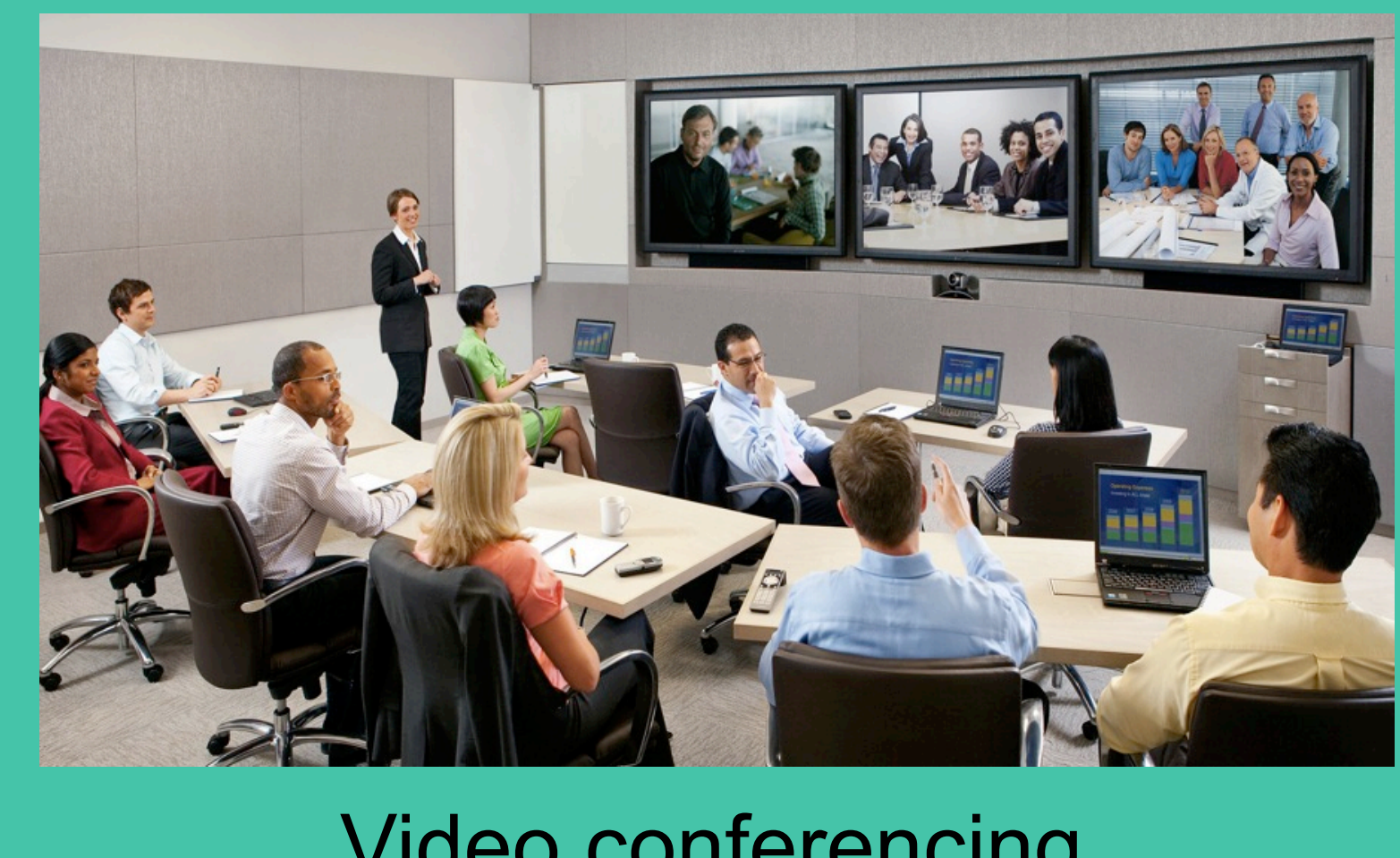

Video conferencing

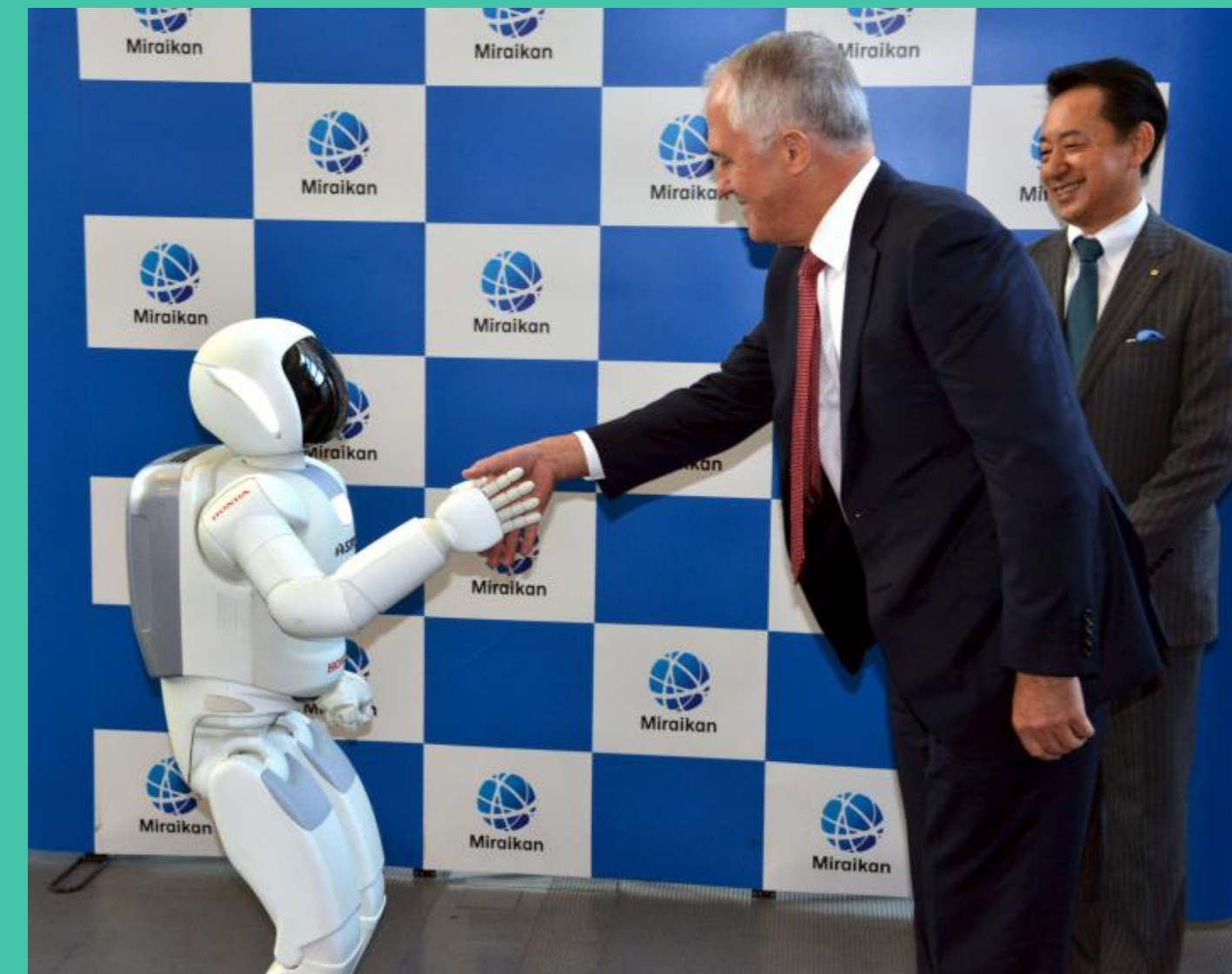

Human - Robot interaction

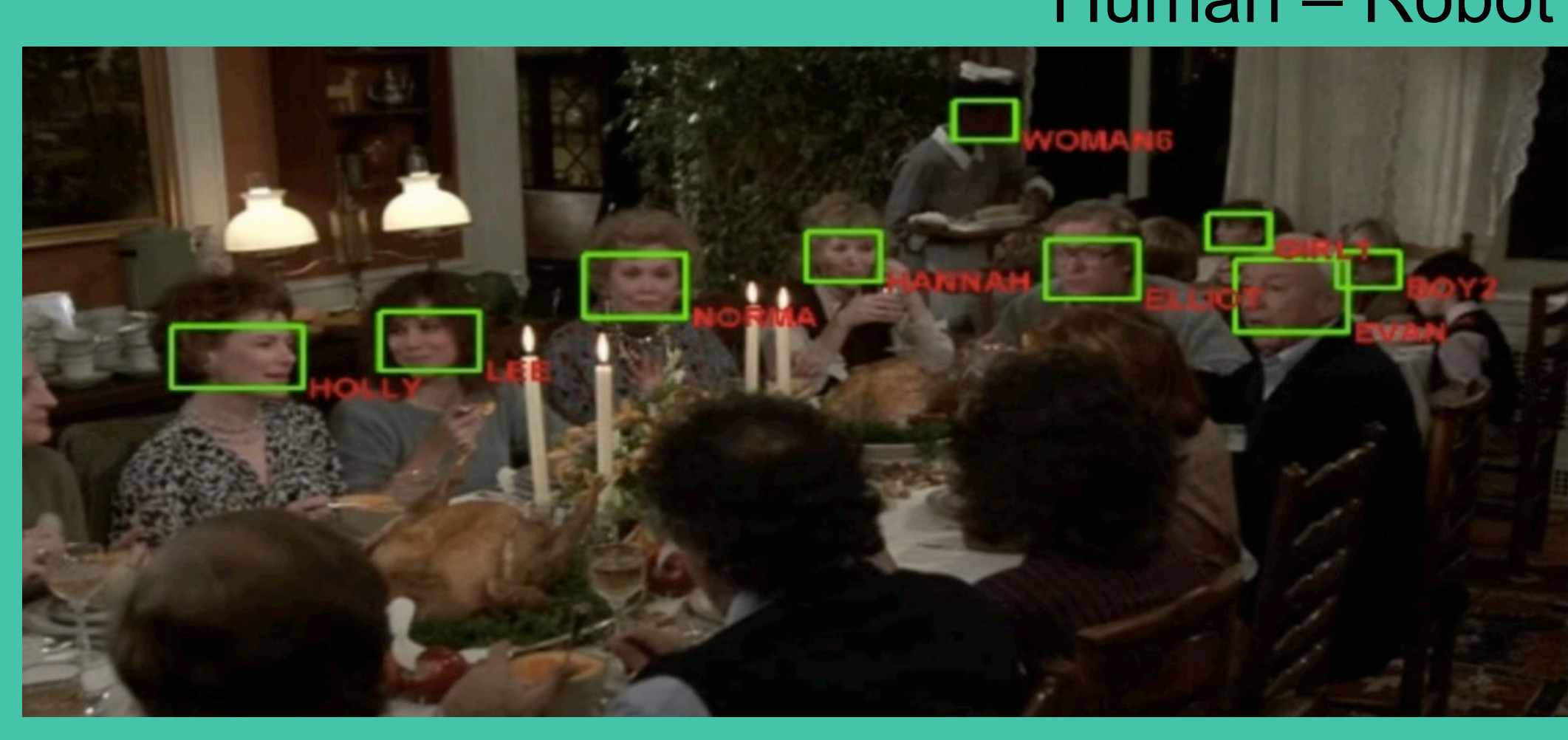

Video diarization

Spatio-temporal Feature Extraction

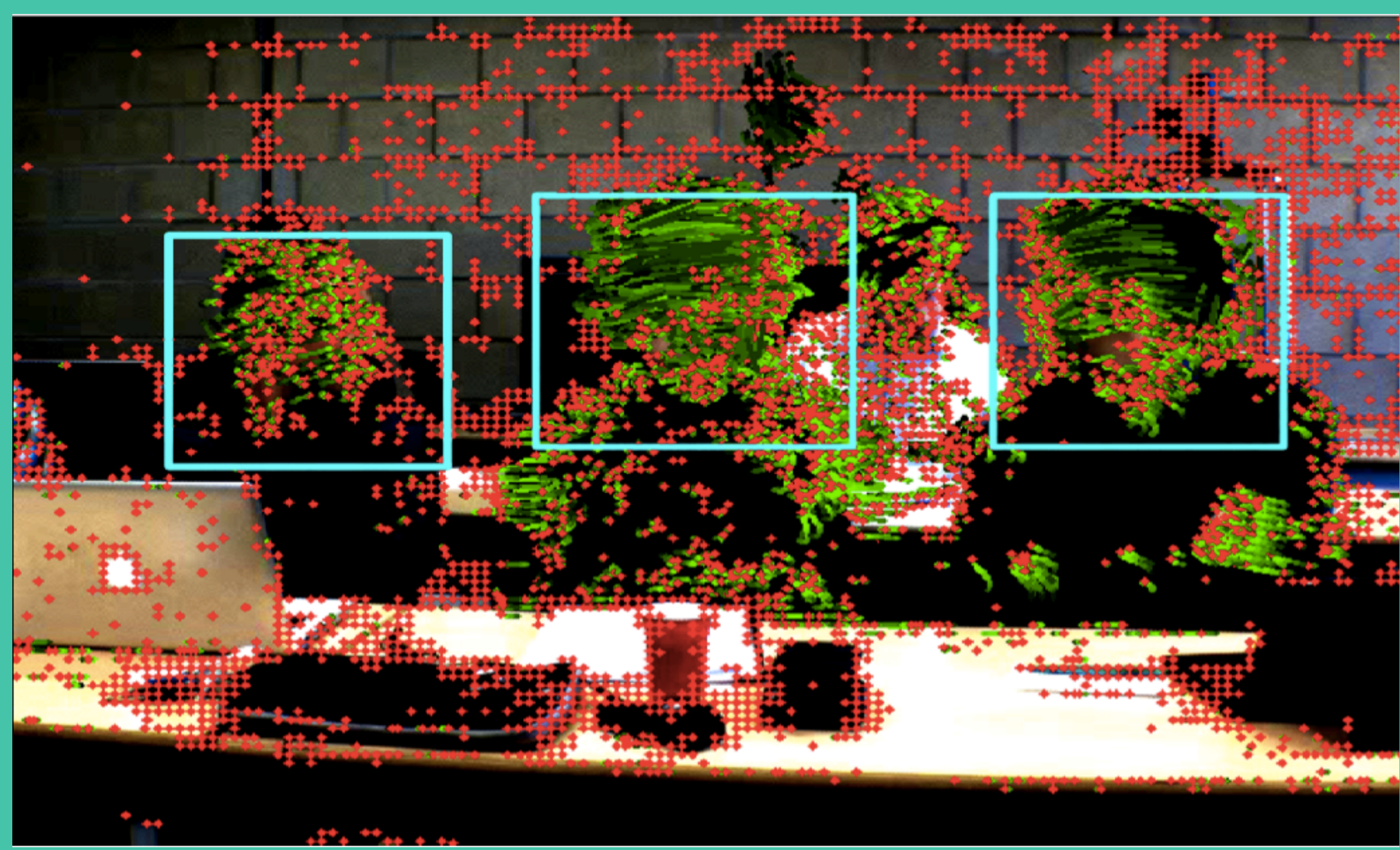

Improved Dense Trajectories (IDT) are extracted from within upper body tracks, pooled with Fisher vectors and used as features for the active speaker classifier

Weak Supervision from Voice Activity Detection

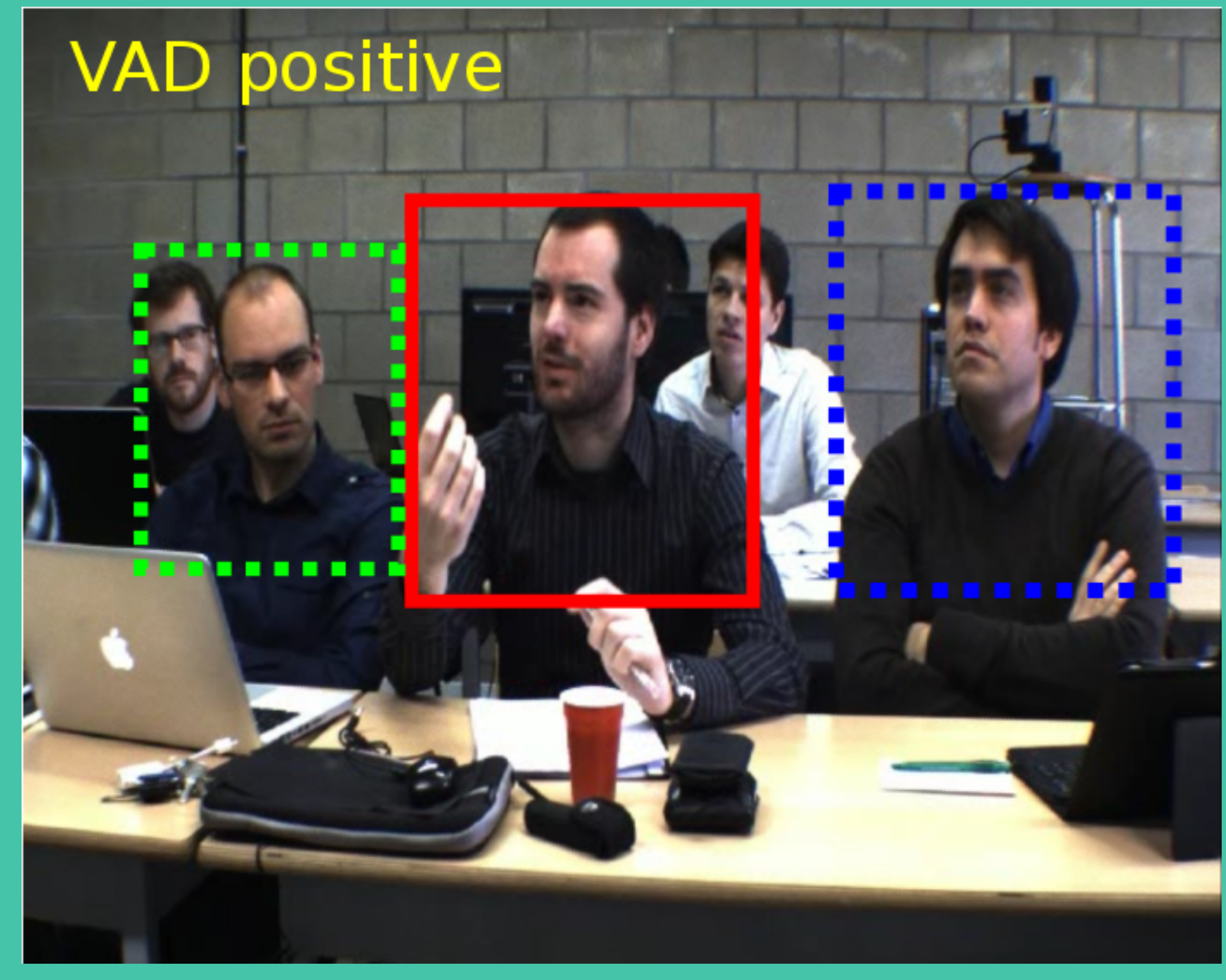

Voice Activity Detection (VAD) based audio supervision tells us someone is speaking. But not who. Structured Output Learning is used to learn a latent SVM Classifier in the presence of partially observed (latent) inputs.
Audio weakly supervises Video Learning
Spatio-temporal feature extraction

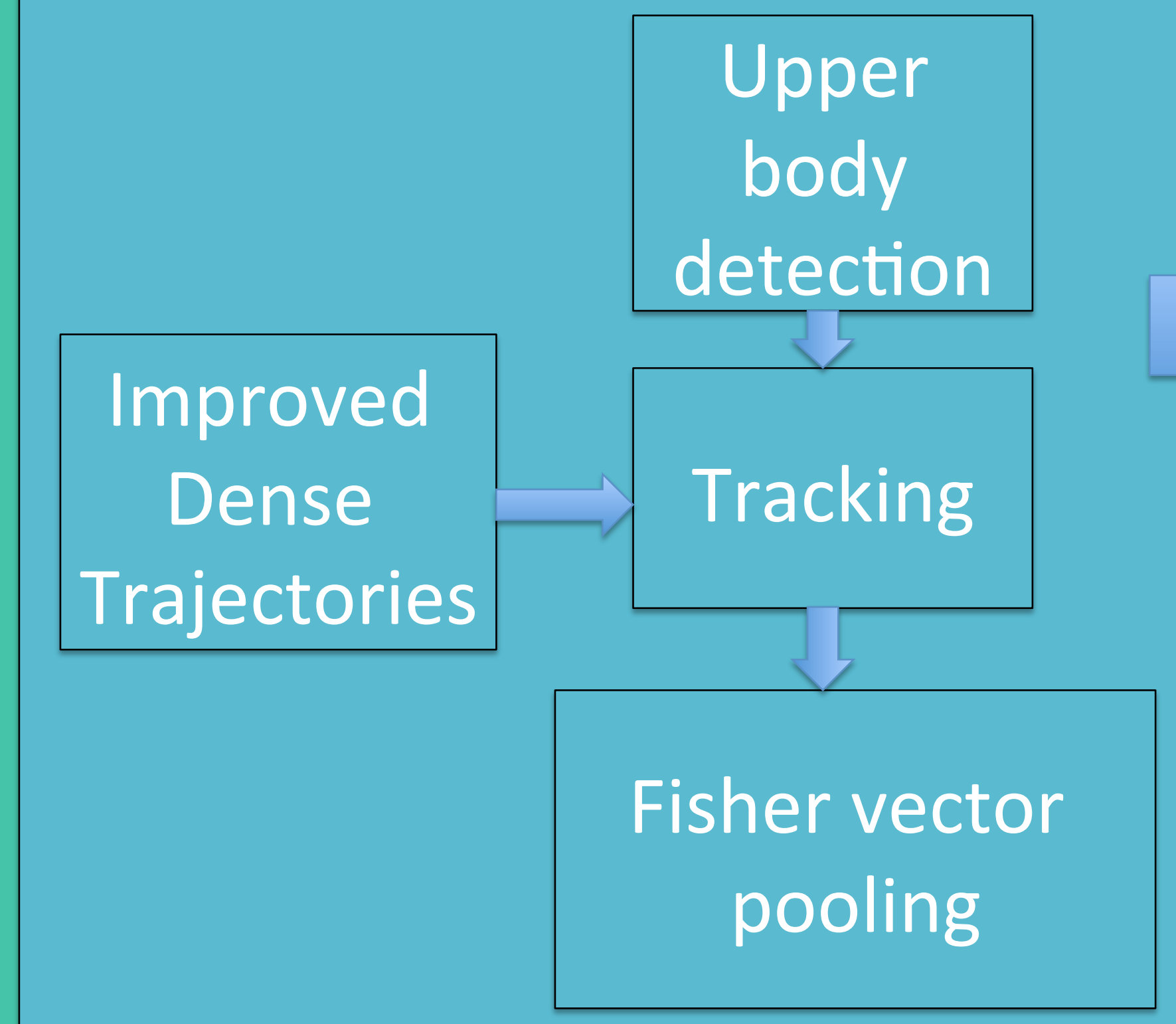

Improvements with Online Learning on the Columbia Dataset

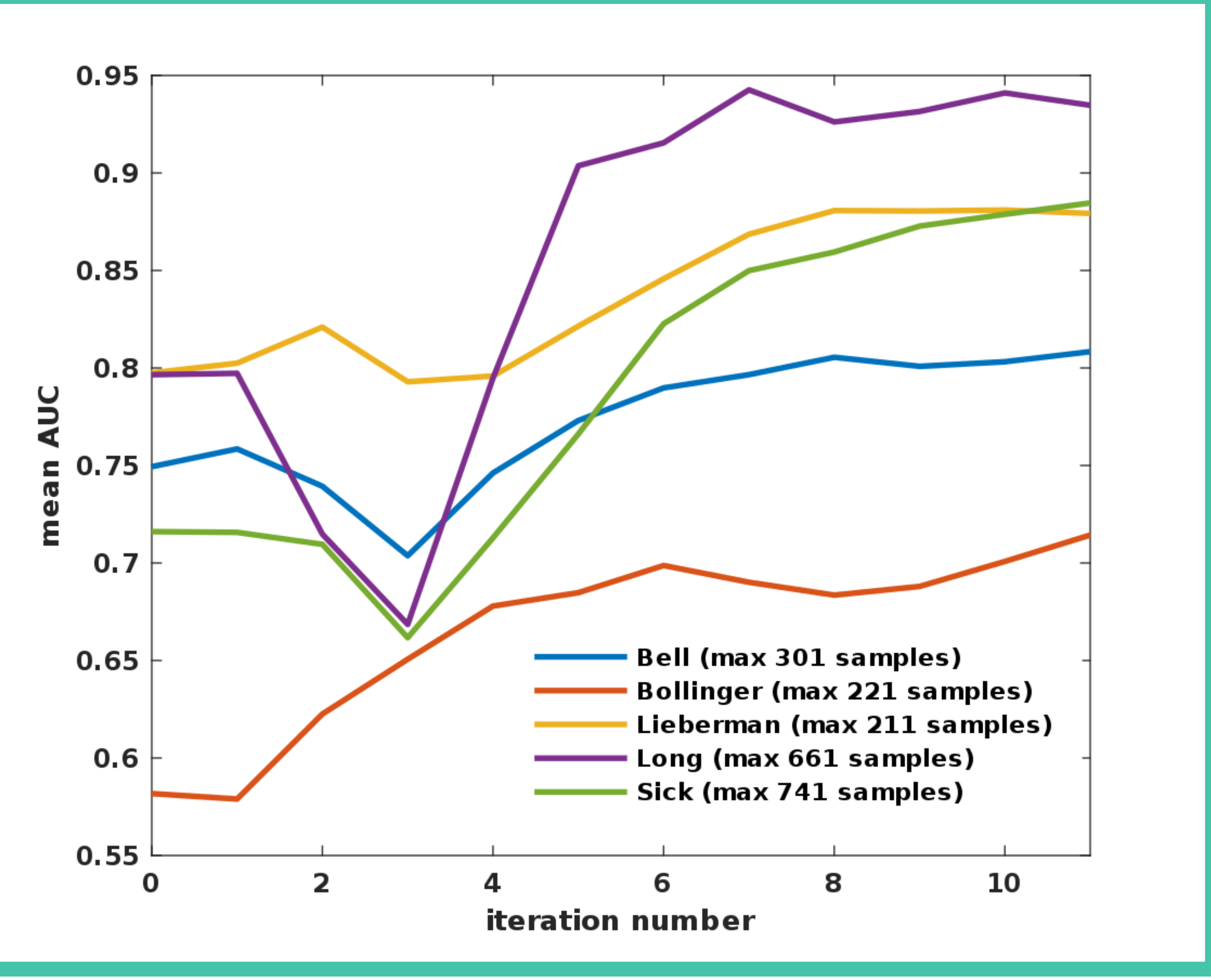

The Columbia dataset is a panel discussion of 5 speakers over 35 minutes. 2-3 people are in frame at any one time, and only one of them is speaking. The generic classifier learnt offline on other data improves with online learning using just a few seconds of video on specific speakers in the Columbia video. Again, audio VAD weakly supervises online learning.
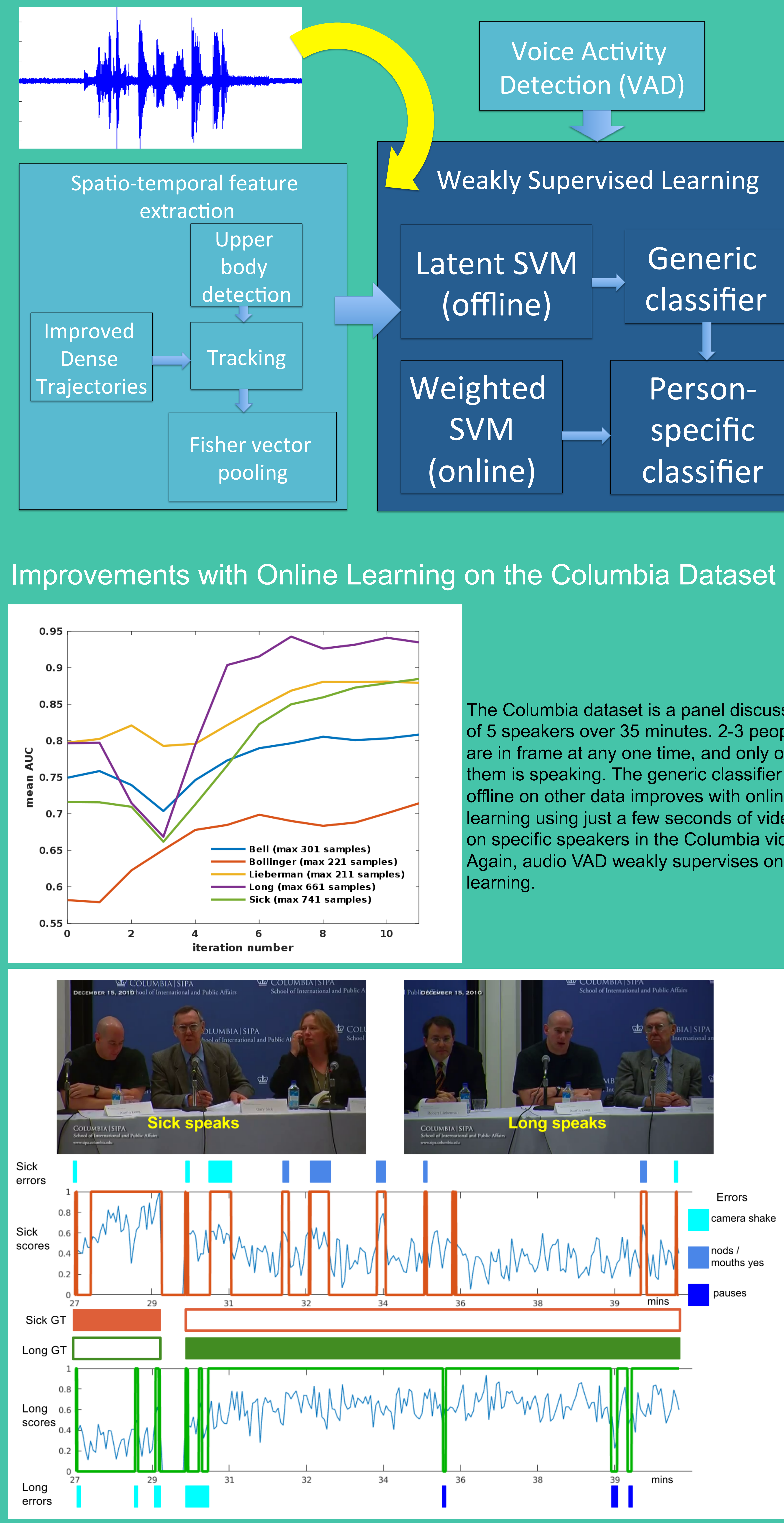

Normalized raw scores (blue) with the online-learnt classifier and thresholded and temporally smoothed speak/non-speak values for speakers Sick (red) and Long (green), along with Ground Truth (GT, solid colour = speak), in minutes 27:00 to 40:00 in the Columbia dataset. 